\title{
Embedding of Large Boolean Functions for Reversible Logic
}

\author{
Mathias Soeken, Member, IEEE, Robert Wille, Member, IEEE, Oliver Keszocze, Student Member, IEEE, \\ D. Michael Miller, Member, IEEE, Rolf Drechsler, Senior Member, IEEE
}

\begin{abstract}
Reversible logic represents the basis for many emerging technologies and has recently been intensively studied. However, most of the Boolean functions of practical interest are irreversible and must be embedded into a reversible function before they can be synthesized. Thus far, an optimal embedding is guaranteed only for small functions, whereas a significant overhead results when large functions are considered. In this paper, we study this issue. We prove that determining an optimal embedding is coNP-hard already for restricted cases. Then, we propose heuristic and exact methods for determining both the number of additional lines as well as a corresponding embedding. For the approaches we considered sums of products and binary decision diagrams as function representations. Experimental evaluations show the applicability of the approaches for large functions. Consequently, the reversible embedding of large functions is enabled as a precursor to subsequent synthesis.
\end{abstract}

\section{INTRODUCTION}

$\mathbf{S}$ YNTHESIS of reversible circuits has been intensively studied in the recent past [4], [8], [9], [16], [19]. Since most Boolean functions of practical interest are irreversible, such functions are embedded into reversible ones prior to synthesis. Given an $m$-output irreversible function $f$ on $n$ variables, a reversible function $g$ with $m+k$ outputs is determined such that $g$ agrees with $f$ on the first $m$ components. The overhead in terms of the $k$ additional variables shall be kept as small as possible. The embedding is called optimal if $k$ is minimal.

Thus far, only synthesis approaches based on truth tables allow for a determination of an optimal embedding. However, determining an efficient embedding for large irreversible functions, i.e. functions with up to a hundred variables, is an open research problem which significantly hindered the development of scalable synthesis approaches for reversible logic.

In this work, we study this issue from both, theoretical and practical, perspectives. First, we derive two lower bounds for determining the minimal value of $k$, namely (1) we show that already when $m=1$ it is coNP-hard to determine if the minimal $k$ equals $n-1$ and (2) we show that, even when $n-m$ is bounded by a constant, it is coNP-hard to decide if the minimal $k$ equals $n-m$. Hence, computing the minimal

This work has been submitted to the IEEE for possible publication. Copyright may be transferred without notice, after which this version may no longer be accessible.

M. Soeken, R. Wille, O. Keszocze, and R. Drechsler are with the University of Bremen, Germany, and the German Research Center for Artificial Intelligence (DFKI).

D. M. Miller is with the University of Victoria, BC, Canada.

M. Soeken is corresponding author: msoeken@cs.uni-bremen.de number $k$ of additional variables is not feasable in polynomial time unless $\mathrm{P}=\mathrm{NP}$.

We then propose algorithms for both heuristic and optimal embeddings and evaluate for which cases an efficient application is possible. We differentiate between (1) determining the required number of additional lines and (2) determining the concrete embedding. The key element in both steps is to use sum-of-product expressions (SOPs) and binary decision diagrams (BDDs) which potentially allow for a more compact function representation compared to truth tables. As a result, an embedding methodology results which can process large irreversible functions for the first time.

While so far efficient embedding of irreversible functionality was restricted to very small functions, the proposed approach enables embedding of functions containing hundreds of variables. We confirm this by comprehensive experimental evaluations.

The contributions described in this paper are as follows:

- We provide lower bounds for the embedding problem.

- We present three algorithms for determining the number of additional lines of large irreversible functions, one heuristic algorithm (cube-based) and two exact ones (cube-based and BDD-based).

- We propose two algorithms for embedding large irreversible functions, i.e. one exact algorithm (cube-based) and one heuristic algorithm (BDD-based) that respects the theoretical upper bound.

- Finally, we provide open source implementations for all presented algorithms.

The paper is organized as follows. Preliminary definitions are given in the next section. Section [III provides the background on the synthesis of reversible function and motivates the problem that is addressed by this work. We present known upper bounds and derive new lower bounds for the problem in Section IV Approaches for approximating and determining the minimal number of additional lines are described in Section $\mathbf{V}$. Afterwards, approaches for exact and heuristic approaches are described in Section VI. Section VII presents and discusses the results from the experimental evaluation before the paper is concluded in Section VIII.

\section{PRELIMINARIES}

In this section we introduce notations. In Section $\amalg-A$ we introduce (reversible) Boolean functions, in Section [I-B we review BDDs, and in Section $\amalg I-C$ we define notations for SOPs. 


\begin{tabular}{cc|ccc}
\hline$x_{1}$ & $x_{2}$ & $y$ & $\gamma_{1}$ & $\gamma_{2}$ \\
\hline $\mathbf{0}$ & $\mathbf{0}$ & $\mathbf{0}$ & 0 & 0 \\
$\mathbf{0}$ & $\mathbf{1}$ & $\mathbf{0}$ & 0 & 1 \\
$\mathbf{1}$ & $\mathbf{0}$ & $\mathbf{0}$ & 1 & 0 \\
$\mathbf{1}$ & $\mathbf{1}$ & $\mathbf{1}$ & 0 & 0 \\
\hline
\end{tabular}

\begin{tabular}{ccc|ccc}
\hline$\kappa$ & $x_{1}$ & $x_{2}$ & $y$ & $\gamma_{1}$ & $\gamma_{2}$ \\
\hline 0 & $\mathbf{0}$ & $\mathbf{0}$ & $\mathbf{0}$ & 0 & 0 \\
0 & $\mathbf{0}$ & $\mathbf{1}$ & $\mathbf{0}$ & 0 & 1 \\
0 & $\mathbf{1}$ & $\mathbf{0}$ & $\mathbf{0}$ & 1 & 0 \\
0 & $\mathbf{1}$ & $\mathbf{1}$ & $\mathbf{1}$ & 0 & 0 \\
1 & 0 & 0 & 0 & 1 & 1 \\
1 & 0 & 1 & 1 & 0 & 1 \\
1 & 1 & 0 & 1 & 1 & 1 \\
1 & 1 & 1 & 1 & 1 & 1 \\
\hline
\end{tabular}

(a) Garbage outputs

(b) Constant input

Fig. 1. Embedding of the AND function

\section{A. Boolean Functions and Reversible Boolean Functions}

Let $\mathbb{B} \stackrel{\text { def }}{=}\{0,1\}$ denote the Boolean values and let

$$
\mathcal{B}_{n, m} \stackrel{\text { def }}{=}\left\{f \mid f: \mathbb{B}^{n} \rightarrow \mathbb{B}^{m}\right\}
$$

be the set of all Boolean functions with $n$ inputs and $m$ outputs, where $m, n \geq 1$. We write $\mathcal{B}_{n} \stackrel{\text { def }}{=} \mathcal{B}_{n, 1}$ for each $n \geq 1$ and assume that each $f \in \mathcal{B}_{n}$ is represented by a propositional formula over the variables $\left\{x_{1}, \ldots, x_{n}\right\}$. Conversely, any $m$-tuple $t$ of Boolean functions over variables $\left\{x_{1}, \ldots, x_{n}\right\}$ corresponds to a unique Boolean function $f_{t} \in \mathcal{B}_{n, m}$. We assume that each function $f \in \mathcal{B}_{n, m}$ is represented as a tuple $f=\left(f_{1}, \ldots, f_{m}\right)$ where $f_{i} \in \mathcal{B}_{n}$ for each $i \in\{1, \ldots, m\}$ and hence $f(\vec{x})=\left(f_{1}(\vec{x}), \ldots, f_{m}(\vec{x})\right)$ for each $\vec{x} \in \mathbb{B}^{n}$.

Given a Boolean function $f \in \mathcal{B}_{n, m}$ the sets on $(f) \stackrel{\text { def }}{=}\{\vec{x} \in$ $\left.\mathbb{B}^{n} \mid f(\vec{x}) \neq 0^{m}\right\}$ and off $(f) \stackrel{\text { def }}{=}\left\{\vec{x} \in \mathbb{B}^{n} \mid f(\vec{x})=0^{m}\right\}$ are called $O N$-set and $O F F$-set of $f$. It can easily be seen that on $(f) \cup \operatorname{off}(f)=\mathbb{B}^{n}$.

Assume $f=\left(f_{1}, \ldots, f_{m}\right) \in \mathcal{B}_{n, m}$ and $g=\left(g_{1}, \ldots, g_{m^{\prime}}\right) \in$ $\mathcal{B}_{n, m^{\prime}}$, where $m^{\prime} \geq m$. We write $f=\left.g\right|_{m}$ in case $f_{i}(\vec{x})=$ $g_{i}(\vec{x})$ for each $\vec{x} \in \mathbb{B}^{n}$ and each $i \in\{1, \ldots, m\}$ and say that $f$ is the $m$-projection of $g$. We say $f \in \mathcal{B}_{n}$ is valid if $f(\vec{x})=1$ for each $\vec{x} \in \mathbb{B}^{n}$.

Given a function $f=\left(f_{1}, \ldots, f_{m}\right) \in \mathcal{B}_{n, m}$ its characteristic function $\chi_{f} \in \mathcal{B}_{n+m}$ is defined as

$$
\chi_{f}(\vec{x}, \vec{y}) \stackrel{\text { def }}{=} \begin{cases}1 & f(\vec{x})=\vec{y} \\ 0 & \text { otherwise }\end{cases}
$$

for each $\vec{x} \in \mathbb{B}^{n}$ and each $\vec{y} \in \mathbb{B}^{m}$. The characteristic function allows one to represent any multiple-output function as a single-output function. It can be computed from a multipleoutput function by adding to the variables $\left\{x_{1}, \ldots, x_{n}\right\}$ the additional output variables $\left\{y_{1}, \ldots, y_{m}\right\}$ :

$$
\bigwedge_{i=1}^{m}\left(y_{i} \leftrightarrow f_{i}\left(x_{1}, \ldots, x_{n}\right)\right)
$$

Given a Boolean function $f \in \mathcal{B}_{n}$ over the variables $X=$ $\left\{x_{1}, \ldots, x_{n}\right\}$ and a variable $x_{i} \in X$, we define the positive co-factor $f_{x_{i}} \in \mathcal{B}_{n-1}$ and the negative co-factor $f_{\bar{x}_{i}} \in$ $\mathcal{B}_{n-1}$ as $f_{x_{i}}=f\left(x_{1}, \ldots, x_{i-1}, 1, x_{i+1}, \ldots, x_{n}\right)$ and $f_{\bar{x}_{i}}=$ $f\left(x_{1}, \ldots, x_{i-1}, 0, x_{i+1}, \ldots, x_{n}\right)$, respectively.

A function $f \in \mathcal{B}_{n, m}$ is called reversible if $f$ is bijective, otherwise it is called irreversible. Clearly, if $f$ is reversible, then $n=m$. A function $g \in \mathcal{B}_{n, m+k}$ embeds $f \in \mathcal{B}_{n, m}$, if $g$ is injective and $\left.f \equiv g\right|_{m}$. The function $g$ is called an embedding and the additional $k$ outputs of $g$ are referred to as garbage outputs and are denoted by $\vec{\gamma}$ later. We are interested in those embeddings of $f$, where $k$ is minimal. Let

$$
\mu(f) \stackrel{\text { def }}{=} \max \left\{\# f^{-1}(\{\vec{y}\}) \mid \vec{y} \in \mathbb{B}^{m}\right\}
$$

denote the number of occurrences of the most frequent output pattern. It is not hard to see that $\ell(f) \stackrel{\text { def }}{=}\left\lceil\log _{2} \mu(f)\right\rceil$ is both an upper and a lower bound (and thus an optimal bound) for $k$. Thus, if $k=\ell(f)$, then the embedding $g$ is called optimal.

Example 1: The AND function $\wedge \in \mathcal{B}_{2}$ can be embedded into a reversible function $g \in \mathcal{B}_{2,3}$ which is illustrated in Fig. 1a The most frequent output pattern is 0 , hence $\mu=3$. The embedding $g$ is optimal.

In order to obtain a reversible function for an embedding $g$, additional input variables might need to be added. Bijectivity can readily be achieved, e.g. by adding additional inputs such that $f$ evaluates to its original values in case these inputs are assigned the constant value 0 and each output pattern that is not in the image of $g$ is arbitrary distributed among the new input patterns. The additional inputs are referred to as constant inputs.

Example 2: A constant input assignment, denoted $\kappa$, for the embedded AND function in Fig. 1a is given in Fig. 1b

Different algorithms that perform an optimal embedding of irreversible functions based on their truth table description have been proposed in the past [11].

\section{B. Binary Decision Diagrams}

Binary Decision Diagrams (BDD) [3] are an established data structure for representing Boolean functions. While the general concepts are briefly outlined in this section, the reader is referred to the literature for a comprehensive overview [3], [7].

Let $X=\left\{x_{1}, \ldots, x_{n}\right\}$ be a set of variables of a Boolean function $f \in \mathcal{B}_{n}$. A BDD representing the function $f$ is a directed acyclic graph $F$ with non-terminal vertices $N$ and terminal vertices $T \subseteq\{\perp, T\}$ where $N \cap T=\emptyset$ and $T \neq$ $\emptyset$. Each non-terminal vertex $v \in N$ is labeled by a variable from $X$ and has exactly two children, low $v$ and high $v$. The directed edges to these children are called low-edge and highedge and are drawn dashed and solid, respectively. A nonterminal vertex $v$ labeled $x_{i}$ represents a function denoted $\sigma(v)$ given by the Shannon decomposition [15]

$$
\sigma(v)=\bar{x}_{i} \sigma(\text { low } v)+x_{i} \sigma(\text { high } v)
$$

where $\sigma(\operatorname{low} v)$ and $\sigma($ high $v)$ are the functions represented by the children of $v$ with $\sigma(\perp) \equiv 0$ and $\sigma(\square) \equiv 1$. The BDD $F$ has a single start vertex $s$ with $\sigma(s) \equiv f$.

A BDD is ordered if the variables of the vertices on every path from the start vertex to a terminal vertex adhere to a specific ordering. Not all of the variables need to appear on a particular path and a variable can appear at most once on any path. A BDD is reduced if there are no two non-terminal vertices representing the same function, hence the representation of common subfunctions is shared. Complemented edges can 


\begin{tabular}{ccccc|ccc}
$x_{1}$ & $x_{2}$ & $x_{3}$ & $x_{4}$ & $x_{5}$ & $y_{1}$ & $y_{2}$ & $y_{3}$ \\
\hline 1 & - & - & 0 & - & 1 & 0 & 0 \\
0 & 0 & - & - & - & 0 & 1 & 0 \\
1 & 1 & - & - & 1 & 0 & 0 & 1 \\
- & 1 & 0 & - & - & 0 & 0 & 1 \\
1 & 0 & - & 1 & - & 1 & 0 & 1 \\
1 & 1 & - & 1 & 0 & 1 & 0 & 1
\end{tabular}

Fig. 2. PLA representation

additionally reduce the size of a BDD and are marked using a solid dot. In the following only reduced, ordered BDDs are considered and for brevity referred to as BDDs.

Multiple-output functions can be represented by a single BDD that has more than one start vertex. Common subfunctions that can be shared among the functions decrease the overall size of the BDD. In fact, many practical Boolean functions can efficiently be represented using BDDs and efficient manipulations and evaluations are possible [3].

\section{Sum-Of-Product Representation}

Each Boolean function $f \in \mathcal{B}_{n}$ can be represented in SumOf-Product (SOP) representation in which $f$ is of the form

$$
f\left(x_{1}, \ldots, x_{n}\right)=\bigvee_{i=1}^{k} x_{1}^{p_{i, 1}} x_{2}^{p_{i, 2}} \cdots x_{n}^{p_{i, n}}
$$

with polarities $p_{i, j} \in\{0,1,2\}$ for $j \in\{1, \ldots, n\}$ and

$$
x^{p}= \begin{cases}\bar{x} & \text { if } p=0 \\ x & \text { if } p=1 \\ 1 & \text { if } p=2\end{cases}
$$

If $p \neq 2, x^{p}$ is called a literal, otherwise $x^{p}$ is referred to as don't care. We call each $c_{i}=x_{1}^{p_{i, 1}} x_{2}^{p_{i, 2}} \cdots x_{n}^{p_{i, n}}$ a cubd of weight

$$
\omega\left(c_{i}\right)=\#\left\{j \mid p_{i, j} \neq 2\right\} .
$$

That is, the weight refers to the number of literals in $c_{i}$. Note that $\#$ on $\left(c_{i}\right)=2^{n-\omega\left(c_{i}\right)}$, i.e. by removing one literal one doubles the number of input assignments that satisfy $c_{i}$. The set

$$
\operatorname{dc}(c)=\left\{x_{i}^{p_{i}} \mid i \in\{1, \ldots, n\}, p_{i}=2\right\}
$$

refers to all variables that are don't care and hence not contained as literal in the cube. One can also represent $f$ by its cubes $\left\{c_{1}, \ldots, c_{k}\right\}$ and we write

$$
f \multimap\left\{c_{1}, \ldots, c_{k}\right\}
$$

where $\multimap$ reads "is constructed of."

A multiple output function $f=\left(f_{1}, \ldots, f_{m}\right) \in \mathcal{B}_{n, m}$ is represented by $m$ such sum-of-product forms, i.e. $f_{i} \multimap C_{i}$ for $i \in\{1, \ldots, m\}$ where each $C_{i}$ is a set of cubes. All cubes of $f$ are then given by

$$
C=\bigcup_{i=1}^{m} C_{i}
$$

${ }^{1}$ Often, also the term monom is used synonymously.
Conversely, we can represent $f$ also as a function that maps each cube from $C$ to those output functions that are constructed from this cube. More formally, $f$ is represented by a function

$$
P_{f}: C \rightarrow \mathcal{P}\left(\left\{f_{1}, \ldots, f_{m}\right\}\right)
$$

where $\mathcal{P}$ denotes the power set and with

$$
P_{f}(c)=\left\{f_{i} \mid c \in C_{i}\right\}
$$

We refer to $P_{f}$ as the PLA representation of $f$. Since the power set of all output functions is being used in several places, we will use $\mathcal{P}(f) \stackrel{\text { def }}{=} \mathcal{P}\left(\left\{f_{1}, \ldots, f_{m}\right\}\right)$ in the remainder for a more compact representation. We will later see that the PLA representation of a function turns out to be convenient to formulate algorithms.

Example 3: The PLA representation of the function $f=$ $\left(f_{1}, f_{2}, f_{3}\right) \in \mathcal{B}_{5,3}$ with

$$
\begin{aligned}
& y_{1}=x_{1} \bar{x}_{4} \vee x_{1} \bar{x}_{2} x_{4} \vee x_{1} x_{2} x_{4} \bar{x}_{5}, \\
& y_{2}=\bar{x}_{1} \bar{x}_{2}, \\
& y_{3}=x_{1} x_{2} x_{5} \vee x_{2} \bar{x}_{3} \vee x_{1} \bar{x}_{2} x_{4} \vee x_{1} x_{2} x_{4} \bar{x}_{5} .
\end{aligned}
$$

is illustrated by the table in Fig. 2 As before, we make use of the convention $y_{i}=f_{i}\left(x_{1}, \ldots, x_{5}\right)$.

Each input cube of $f$ is represented by its polarities with the exception that we write - instead of 2 . For each output function we write 1 , if the corresponding cube is in its constructing set, otherwise 0 .

The table shall not be confused with a truth table, in particular the 0's in the output columns do not indicate that the functions evaluate to 0 for the corresponding input cube. As an example, we have $f_{1}\left(x_{1}, x_{2}, \bar{x}_{3}, x_{4}, \bar{x}_{5}\right)=1$ due to the sixth cube. However, this input pattern is also contained in the fourth cube which is not in the constructing set of $f_{1}$.

\section{Synthesis of Reversible Functions}

Reversible circuits on $r$ lines composed of special reversible gates, e.g. Toffoli gates, represent reversible functions of $r$ variables. In turn, every reversible function can be realized with a reversible circuit. The problem of finding a reversible circuit for a given function is called synthesis and, since most of the functions of practical interest are irreversible, we are considering the following synthesis problem in this paper: Given an arbitrary Boolean function $f \in \mathcal{B}_{n, m}$, a reversible circuit should be determined that represents $g \in \mathcal{B}_{r, r}$ such that $g$ embeds $f$.

The synthesis of reversible functions has been an intensively studied research area in the last decade. Initially, algorithms based on truth table representations have been proposed [5], [9]. Due to their non-scalable representation, none of these is capable of efficiently synthesizing functions with more than 20 variables. This fact is reflected in the first two rows in Table [1] The $R$ in the respective rows denotes that these approaches are only directly applicable for reversible functions. However, irreversible functions are handled by embedding them first as described in the previous section. Moreover, an 
TABLE I

SYNTHESIS OF REVERSIBLE FUNCTIONS

\begin{tabular}{llccc}
\hline Representation & S & Scalability & E & Overhead \\
\hline Truth table (exact) & $R$ & $\leq 7$ variables & y & minimal \\
Truth table (heuristic) & $R$ & $\leq 20$ variables & y & minimal \\
Symbolic & $I$ & $\approx 100$ variables & N/A & large \\
Symbolic (QMDDs) & $R$ & $\approx 100$ variables & $\mathrm{n}$ & minimal \\
\hline
\end{tabular}

S: Supports reversible (R) or also irreversible (I) functions

E: Embedding approaches are available (y) or not (n)

optimal embedding and therefore a minimal overhead in terms of additional variables can be guaranteed since the value of $\ell(f)$ can readily be determined from the truth table, in other words $r=m+\ell(f)$.

In order to synthesize larger functions, researchers have been investigating the use of symbolic representations. An approach based on BDDs [20] is one of the first solutions able to synthesize functions with more than one hundred variables (as summarized in the third row of Table 【). In particular, by using BDDs it is also possible to directly start with the irreversible function representation as the embedded takes place implicitly during synthesis (denoted by $I$ in the second column of Table \). However, the newly achieved scalability is traded off against a large number of additional variables which is much larger than the upper bound [21], in other words $r \gg m+n$.

The large number of additional variables results from the fact that this algorithm performs synthesis in a hierarchical fashion rather than considering the function as a whole. Determining an optimal embedding similar to the truth tablebased approaches is not applicable. Although optimization approaches exist that reduce additional variables in a postsynthesis step [22], a satisfying result can rarely be achieved as evaluated in [21].

Following these considerations, an alternative has been proposed in [19] that exploits another symbolic representation and relies on reversible functions. For this purpose, Quantum Multiple-values Decision Diagrams QMDDs [10] are utilized which are data-structures particularly suited for the representation of reversible functions. For the first time, this enabled the synthesis of reversible functions with up to 100 variables and without adding any additional variables. However, this algorithm is of not much help, since so far it was unknown how to embed the given irreversible function into a QMDD representing the reversible embedding (as summarized in the forth row of Table @).

In summary, synthesis of reversible circuits for irreversible functions always requires an efficient embedding. For small functions, this is no problem and in fact, optimal embeddings can readily be obtained. In contrast, if larger functions are addressed, previous solutions led to significantly high overhead. Although scalable synthesis methods for large reversible functions are available, how to derive the respective embeddings is unknown so far.

In this paper, we are addressing this issue by lifting embedding from truth table based approaches to symbolic ones. For this purpose, we are exploiting observations by Bennett on upper bounds and general embeddings. Additionally, we use the symbolic representation of BDDs. However, we first consider the theoretical complexity of the embedding problem.

\section{BOUndS FOR THE Number of AdDitional Lines}

\section{A. Upper Bound}

An upper bound for the number of lines can easily be determined as described by the following proposition.

Proposition 1: Given a function $f \in \mathcal{B}_{n, m}$. Then at most $\ell(f)=n$ additional lines are required to embed $f$.

Proof: The value of $\mu(f)$ is maximized if there exists one $\vec{y} \in \mathbb{B}^{m}$ such that for all $\vec{x} \in \mathbb{B}^{n}$ we have $f(\vec{x})=\vec{y}$. In this case $\mu(f)=2^{n}$. Hence, $\ell(f)=\left\lceil\log _{2} 2^{n}\right\rceil=n$

\section{B. Lower Bounds}

In this section, we concern ourselves with the following question: Given a Boolean function $f \in \mathcal{B}_{n, m}$ and some $\ell \geq 0$, does $\ell=\ell(f)$ hold? We prove two coNP lower bounds in this section, one even when $m$ is a fixed and one even when $\ell$ is fixed.

It is clear that we cannot hope for an efficient procedure for computing an optimal embedding for a given $f \in \mathcal{B}_{n, m}$ if we cannot even compute $\ell(f)$ efficiently.

Proposition 2: For every fixed $m \geq 1$ it is coNP-hard to decide, given $f \in \mathcal{B}_{n, m}$ with $n \geq m$, whether $\ell(f)=n-m$.

Proof: We give a polynomial time many-one reduction from the validity problem, i.e. to decide for a given propositional formula whether it is valid, a coNP-complete problem. Let $\varphi$ be a propositional formula over the variables $\left\{x_{1}, \ldots, x_{j}\right\}$. We put $n \stackrel{\text { def }}{=} j+m$. We will compute in polynomial time an $m$-tuple $t=\left(\psi_{1}, \ldots, \psi_{m}\right)$ of propositional formulas over the variables $\left\{x_{1}, \ldots, x_{j+m}\right\}$ such that $\varphi$ is valid if, and only if, $\ell\left(f_{t}\right)=n-m=j$. We put

$$
\psi_{i}\left(x_{1}, \ldots, x_{j+m}\right) \stackrel{\text { def }}{=} \quad x_{j+i} \wedge \varphi\left(x_{1}, \ldots, x_{j}\right)
$$

for each $i \in\{1, \ldots, m\}$. The correctness of the reduction follows immediately from the following equivalences:

$$
\begin{array}{ll} 
& \varphi \text { is valid } \\
\Leftrightarrow \quad \#\left\{\vec{x} \in \mathbb{B}^{j} \mid \varphi(\vec{x})=1\right\}=2^{j} \\
\stackrel{11}{\Leftrightarrow} \quad \forall \vec{y} \in f_{t}\left(\mathbb{B}^{j+m}\right): \#\left\{\vec{x} \in \mathbb{B}^{j+m} \mid f_{t}(\vec{x})=\vec{y}\right\}=\frac{2^{j+m}}{2^{m}} \\
\Leftrightarrow \quad \mu\left(f_{t}\right)=2^{j} \\
\Leftrightarrow \quad \ell\left(f_{t}\right)=j=n-m
\end{array}
$$

Looking at the proof of Proposition 2 we note that we required $\ell$ to be part of the input for the lower bound to work since we fixed $m$. Moreover the lower bound already holds when $m=1$ and thus when $\ell=n-1$. The dual question arises whether a lower bound can be proven when $\ell$ is a fixed constant. Indeed, we prove that computing $\ell(f)$ is already coNP-hard for every fixed $\ell \geq 0$ (i.e. the input to the problem only consists of $f$ and the value $\ell$ is treated as a fixed constant).

Proposition 3: For each fixed $\ell \geq 0$ it is coNP-hard to decide for a given $f \in \mathcal{B}_{n, m}$ whether $\ell(f)=\ell$. 
Proof: We give a polynomial time many-one reduction from the validity problem for propositional formulas. For simplicity we only give the proof for $\ell=0$. The case $\ell>0$ works completely analogously. Fix a propositional formula $\varphi \in \mathcal{B}_{n}$ over the variables $\left\{x_{1}, \ldots x_{n}\right\}$. We will compute in polynomial time a function $f=\left(f_{1}, \ldots, f_{n}\right)$, where each $f_{i}$ is presented by a propositional formula $\varphi_{i}$ over the variables $\left\{x_{1}, \ldots, x_{n}\right\}$ such that $\varphi$ is valid if, and only if, $f$ is injective. We set

$$
\varphi_{i}\left(x_{1}, \ldots, x_{n}\right) \stackrel{\text { def }}{=} x_{i} \wedge \varphi\left(x_{1}, \ldots, x_{n}\right) \wedge \varphi(0, \ldots, 0)
$$

for each $i \in\{1, \ldots, n\}$.

For correctness, we have to show that $\varphi$ is valid if, and only if, $\ell(f)=0$, which in turn is equivalent to $f$ being injective.

Let us first assume that $\varphi$ is valid. Then $\varphi_{i}$ is logically equivalent to $x_{i}$ for each $i \in\{1, \ldots, n\}$ by definition (12). Thus, $f$ is equivalent to the identity $\operatorname{id}_{n} \in \mathcal{B}_{n, n}$, i.e. $\operatorname{id}_{n}(\vec{x}) \stackrel{\text { def }}{=}$ $\vec{x}$ for each $\vec{x} \in \mathbb{B}^{n}$.

Conversely, assume that $\varphi$ is not valid. Then there exists some $\vec{x} \in \mathbb{B}^{n}$ with $\varphi(\vec{x})=0$. We make a case distinction. In case $\vec{x}=0^{n}$, we immediately have that $\varphi_{i}$ is logically equivalent to 0 and thus $f$ is not injective. Now assume that $\vec{x} \neq 0^{n}$. Since $\varphi\left(0^{n}\right)=0^{n}$ and $\varphi(\vec{x})=0^{n}$ and also $\vec{x} \neq 0^{n}$, the function $f$ is not injective.

We note that two simple lower bound corollaries follow from Proposition 2 and 3 . (1) it is coNP-hard to decide if for a given function $f \in \mathcal{B}_{n, n}$ and a given $\ell \geq 0$ whether there exists an injective $g \in \mathcal{B}_{n, n+\ell}$ that embeds $f$, (2) there exists no polynomial time algorithm that computes, given a function $f \in \mathcal{B}_{n, n}$, the minimal $\ell \geq 0$ such that $f$ can be embedded by some function $g \in \mathcal{B}_{n, n+\ell}$ unless $\mathrm{P}=\mathrm{NP}$.

\section{Determining the Number of Additional Lines}

In this section we propose three algorithms to determine the number of additional lines. The first algorithm approximates the number of lines while the second and third one determine the minimal number.

\section{A. Heuristic Cube-based Approach}

The first approach approximates the number of cubes and is based on the PLA representation of a multiple output function. The approach is an extension of an algorithm presented in [212. Given a function, one can approximate the minimal number of additional variables that are needed to embed the function using the following algorithm.

Algorithm H (Heuristic Cube-based Approach). This algorithm approximates $\mu(f)$ by $\hat{\mu}(f)$ for a given function $f \in$ $\mathcal{B}_{n, m}$ given in PLA representation $P_{f}: C \mapsto \mathcal{P}(f)$. An auxiliary array $\mathrm{MU}[o]$ for $o \in \mathcal{P}(f)$ is used to compute possible candidates for $\hat{\mu}(f)$.

H1. [Initialize.] Set $\mathrm{MU}[o] \leftarrow 0$ for each $o \in \mathcal{P}(f)$.

H2. [Loop over $C$ and update MU.] For each $c \in C$, set $\mathrm{MU}\left[P_{f}(c)\right] \leftarrow \operatorname{MU}\left[P_{f}(c)\right]+\# \mathrm{on}(c)$.

\footnotetext{
${ }^{2}$ In [21], the algorithm does not consider the OFF-set of the function and hence, may return an under approximation.
}

\begin{tabular}{ccccc|cccc}
$x_{1}$ & $x_{2}$ & $x_{3}$ & $x_{4}$ & $x_{5}$ & $y_{1}$ & $y_{2}$ & $y_{3}$ & \\
\cline { 1 - 2 } & - & - & 0 & - & 1 & 0 & 0 & 8 \\
0 & 0 & - & - & - & 0 & 1 & 0 & 8 \\
1 & 1 & - & - & 1 & 0 & 0 & 1 & 4 \\
- & 1 & 0 & - & - & 0 & 0 & 1 & 8 \\
1 & 0 & - & 1 & - & 1 & 0 & 1 & 4 \\
1 & 1 & - & 1 & 0 & 1 & 0 & 1 & 2
\end{tabular}$>12$

Fig. 3. PLA representation of a Boolean function

H3. [Count off $(f)$ in MU.] Set MU $[\emptyset] \leftarrow \#$ off $(f)$.

H4. [Determine $\hat{\mu}(f)$.] Set

$$
\hat{\mu}(f) \leftarrow \max \{\operatorname{MU}[o] \mid o \in \mathcal{P}(f)\} .
$$

Remark 1: Although the set $\mathcal{P}(f)$ is exponentially large, Algorithm $\mathrm{H}$ can be efficiently implemented, since it only needs to consider those elements that are in the image of $P_{f}$. Step 3 can be implemented using BDDs.

Example 4: Consider the function given in Fig. 3 Algorithm $\mathrm{H}$ basically assigns the number of input patterns that are represented by an input cube to each line of the PLA representation. In order to approximate, we assume that the $0 \mathrm{~s}$ in the table are part of the output pattern. Values of lines with the same output pattern are added. The OFF-set of the function is not mentioned in the table representation and can be described by the cube 011-- that corresponds to 4 input pattern. The algorithm computes, that approximately $4+8=12$ input patterns map to the output pattern 001 which corresponds to the computed approximation for $\mu(f)$ obtained from Algorithm H. Hence, $3+\left\lceil\log _{2} 12\right\rceil=7$ lines may be sufficient to realize this function as a reversible circuit.

The determined value for $\hat{\mu}(f)$ is still an approximation, since overlaps of the input cubes are not yet considered. For example, the two input cubes discussed in Example 4 share some equal input patterns, i.e. the determined number of 12 occurrences for the output pattern 001 is an approximation. In fact the output patterns that are assumed for the approximation may have nothing in common with the real output patterns of the function that is described by the PLA representation. Consequently, $\hat{\mu}(f)$ can be smaller than $\mu(f)$. An example for this case is given in the following section. However, the results from the experimental evaluation presented in Section VII will show that the approximation is often close to the exact value.

\section{B. Exact Cube-based Approach}

If we had a PLA representation $P_{f}^{\prime}: C^{\prime} \rightarrow \mathcal{P}(f)$ in which no input cube overlaps, i.e. on $\left(c_{i}\right) \cap$ on $\left(c_{j}\right)=\emptyset$ for all pairwise $c_{i}, c_{j} \in C^{\prime}$, one can guarantee that $\hat{\mu}(f)=\mu(f)$ after applying Algorithm $\mathrm{H}$ to $P_{f}^{\prime}$. The expressions represented by such PLAs are called disjoint sum-of-products (DSOP) in the literature and several algorithms to derive such a representation have been described in the past. The most recent results can be found e.g. in [2], [14]. We create a DSOP representation based on an algorithm described in [21] that particularly addresses multiple-output functions.

Please note that the compact representation of a PLA representation highly depends on overlapping input cubes. As 


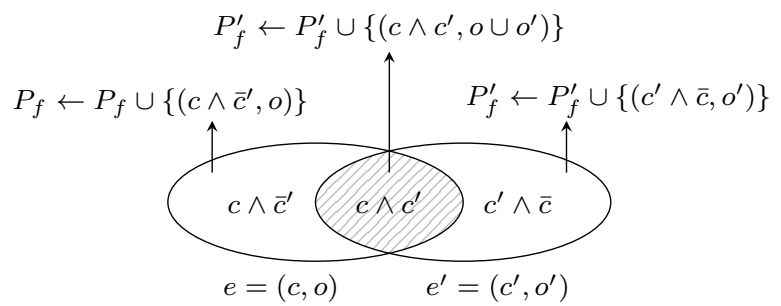

Fig. 4. Illustration for step 3 in Algorithm D

a result, the PLA representation of the DSOP expression is possibly very large. In the worst case, the whole truth table is reconstructed.

Algorithm D (Disjoint Sum-of-Product Computation). Given a PLA representation $P_{f}: C \rightarrow \mathcal{P}(f)$ of a function $f \in \mathcal{B}_{n, m}$, this algorithm computes a new PLA representation $P_{f}^{\prime}: C^{\prime} \rightarrow$ $\mathcal{P}(f)$ where no input cubes overlap, i.e. on $\left(c_{i}\right) \cap$ on $\left(c_{j}\right)=\emptyset$ for all pairwise different $c_{i}, c_{j} \in C^{\prime}$. In the algorithm, $P_{f}$ and $P_{f}^{\prime}$ are treated as mutable relations.

D1. [Terminate?] If $P_{f}=\emptyset$, terminate.

D2. [Pick an entry from $P_{f}$ and iterate over $P_{f}^{\prime}$.] Pick and remove one entry $e=(c, o)$ from $P_{f}$, i.e. set $P_{f} \leftarrow P_{f} \backslash$ $\{e\}$. If there exists an overlapping cube $e^{\prime}=\left(c^{\prime}, o^{\prime}\right) \in P_{f}^{\prime}$, i.e.

$$
c \wedge c^{\prime} \neq \perp
$$

perform step 3. If no such $e^{\prime}$ exists, set $P_{f}^{\prime} \leftarrow P_{f}^{\prime} \cup\{e\}$ and return to step 1 .

D3. [Update $P_{f}$ and $P_{f}^{\prime}$.] Remove the entry $e^{\prime}$ from $P_{f}^{\prime}$, i.e. set $P_{f}^{\prime} \leftarrow P_{f}^{\prime} \backslash\left\{e^{\prime}\right\}$. Keep one "remaining part" for $P_{f}$, i.e.

$$
P_{f} \leftarrow P_{f} \cup\left\{\left(c \wedge \bar{c}^{\prime}, o\right)\right\}
$$

and the other one for $P_{f}^{\prime}$, i.e.

$$
P_{f}^{\prime} \leftarrow P_{f}^{\prime} \cup\left\{\left(c^{\prime} \wedge \bar{c}, o^{\prime}\right)\right\} .
$$

Add the intersection to $P_{f}^{\prime}$ by combining the output sets, i.e.

$$
P_{f}^{\prime} \leftarrow P_{f}^{\prime} \cup\left\{\left(c \wedge c^{\prime}, o \cup o^{\prime}\right)\right\}
$$

Algorithm D transforms the initial PLA representation $P_{f}$ into the initial empty PLA representation $P_{f}^{\prime}$ which represents the same function as DSOP expression. As long $P_{f}$ is non-empty an entry $e=(c, o)$ is chosen for which the following case distinction is applied. If $c$ does not intersect with any other input cube in $P_{f}^{\prime}$, the entry $e$ is removed from $P_{f}$ and directly added to $P_{f}^{\prime}$. Otherwise, i.e. if there exists an entry $e^{\prime}=\left(c^{\prime}, o^{\prime}\right)$ with $c \wedge c^{\prime} \neq \perp$, step 3 is performed. What happens in this step after $e^{\prime}$ has been removed from $P_{f}^{\prime}$ is best illustrated by means of Fig. 4 The ON-set of $c \vee c^{\prime}$ is partitioned into three parts. The part of $c$ that does not intersect $c^{\prime}$ remains in $P_{f}$, and analogously, the part of $c^{\prime}$ that does not intersect $c$ remains in $P_{f}^{\prime}$. The intersection is also added to $P_{f}^{\prime}$, however, the corresponding output functions are combined.

Example 5: An example application of Algorithm D is demonstrated in Fig. 5based on the PLA of Fig. 2. Clearly, the first cube can be moved without merging since $P_{f}^{\prime}$ is initially

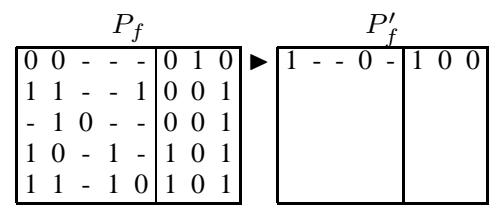

(a) Moving first cube

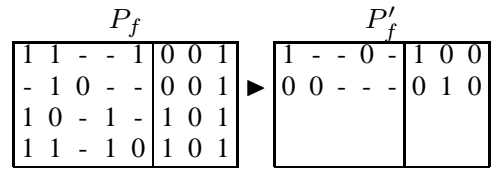

(b) Moving second cube

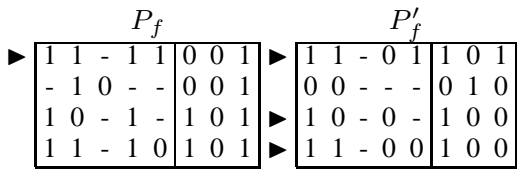

(c) Merging third cube

Fig. 5. DSOP computation for the function in Fig. 3

empty. Affected cubes in steps are marked by ' $\square$ '. Also the second cube can be moved without merging since it does not intersect the first one. The third cube to be moved from $P_{f}$ to $P_{f}^{\prime}$ has the input pattern $c=x_{1} x_{2} x_{5}$. It intersects with the current first cube in $P_{f}^{\prime}$ which has the input pattern $c^{\prime}=x_{1} \bar{x}_{4}$. The "remaining part" for $P_{f}$ is

$$
x_{1} x_{2} x_{5} \wedge \overline{x_{1} \bar{x}_{4}}=x_{1} x_{2} x_{4} x_{5} .
$$

Analogously we have

$$
\overline{x_{1} x_{2} x_{5}} \wedge x_{1} \bar{x}_{4}=x_{1} \bar{x}_{2} \bar{x}_{4} \vee x_{1} x_{2} \bar{x}_{4} \bar{x}_{5}
$$

which yields two new entries to be added to $P_{f}^{\prime}$. The current entry is updated by the intersection

$$
x_{1} x_{2} x_{5} \wedge x_{1} \bar{x}_{4}=x_{1} x_{2} \bar{x}_{4} x_{5}
$$

for which the corresponding output functions are merged. All other steps are not shown explicitly. The final PLA representation for $P_{f}^{\prime}$ is given in Fig. 5(d).

Correctness and completeness: We now prove that Algorithm D is sound and complete.

Lemma 1: Algorithm D is complete.

Proof: We show that the algorithm terminates for every input function $f$. For this purpose, we show that in every iteration the size of the ON-set of the function represented by $P_{f}$ decreases. Consequently, we eventually have $P_{f}=\emptyset$ which leads to termination in step 1 . We perform a case distinction on whether a cube $e^{\prime}$ exists in step 2 .

If such a cube $e^{\prime}$ does not exist, $e=(c, o)$ is removed from $P_{f}$ and we have $c \neq \perp$. Further, nothing is added to $P_{f}$ in this case.

Otherwise, first $c$ is removed from the input cubes and afterwards $c \wedge \bar{c}^{\prime}$ is added. However, since by assumption $c \wedge c^{\prime} \neq \perp$, we have $\#$ on $\left(c \wedge \bar{c}^{\prime}\right)<\#$ on $(c)$.

Lemma 2: Algorithm D is sound.

Proof: Clearly, $P_{f}^{\prime}$ does not contain overlapping input cubes due to step 3 . We now show that in step 1 the function represented by $P_{f} \cup P_{f}^{\prime}$ equals $f$. Since eventually $P_{f}=\emptyset$, 


\begin{tabular}{|c|c|c|c|c|c|}
\hline \multicolumn{6}{|c|}{$P_{f}^{\prime}$} \\
\hline 0 & & & - & & $\begin{array}{|lll|}0 & 1 & 0\end{array}$ \\
\hline J & 1 & & - & & $\begin{array}{lll}0 & 0 & 1\end{array}$ \\
\hline 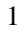 & & & 0 & & $\begin{array}{lll}1 & 0 & 0\end{array}$ \\
\hline ] & & & 1 & & $\begin{array}{lll}1 & 0 & 1\end{array}$ \\
\hline 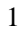 & 1 & & 1 & & $\begin{array}{lll}0 & 0 & 1\end{array}$ \\
\hline 1 & 1 & 0 & 0 & & $\begin{array}{lll}1 & 0 & 1\end{array}$ \\
\hline 1 & 1 & & 1 & & $\begin{array}{lll}1 & 0 & 1\end{array}$ \\
\hline & 1 & 1 & 0 & & $\begin{array}{lll}1 & 0 & 0\end{array}$ \\
\hline & & & 0 & & $\begin{array}{lll}1 & 0 & 1\end{array}$ \\
\hline 1 & & 1 & 1 & & $\begin{array}{lll}1 & 0 & 1\end{array}$ \\
\hline
\end{tabular}

Fig. 6. PLA representation after post compaction

$P_{f}^{\prime}$ represents $f$. We perform a case distinction on whether a cube $e^{\prime}$ exists in step 2 .

If such a cube $e^{\prime}$ does not exist, the case is trivial, since $e$ is removed from $P_{f}$ and directly added to $P_{f}^{\prime}$. For the case that a cube $e^{\prime}$ does exist one can readily observe based on the partition in Fig. 4 that the $\mathrm{ON}$-set of $P_{f} \cup P_{f}^{\prime}$ does not change. Since the output functions are combined for the intersection $c \wedge$ $c^{\prime}$, also the functional semantics of $f$ is preserved.

Post compaction: It is possible to compact the resulting PLA representation that is returned by Algorithm D. For this purpose we create a BDD for each occurring output pattern from the input cubes. This step is only sound because the input cubes do not overlap. By traversing all paths in the BDD we can obtain a new PLA representation for each output cube. It turns out that the PLA representation for the input cubes is more compact compared to the one that resulted from applying Algorithm D.

Example 6: The application of Algorithm D in Example 5 yields a PLA representation that consists of 12 cubes. Applying post compaction as post process yields a PLA representation that consists of 10 cubes (cf. Fig. 6). In the experimental evaluation we will demonstrate that much higher compression can be achieved with this method.

An under approximating example: As discussed in Section $\mathrm{V}$-A Algorithm $\mathrm{H}$ can yield an under-approximation for $\mu(f)$. Consider the following PLA representation for a function with 5 input variables, 3 output variables, and 6 monoms:

$$
\begin{array}{|lllll|lll|}
\hline 0 & 0 & - & 1 & - & 0 & 0 & 1 \\
0 & 0 & 0 & 1 & 0 & 0 & 0 & 1 \\
1 & 1 & 1 & 1 & - & 0 & 0 & 1 \\
- & - & - & 1 & - & 0 & 1 & 0 \\
1 & - & - & - & 1 & 0 & 1 & 1 \\
1 & - & - & - & 0 & 0 & 1 & 1 \\
\hline
\end{array}
$$

Algorithm $\mathrm{H}$ will compute the following output pattern occurrences:

$$
000 \mapsto 8 \quad 001 \mapsto 7 \quad 010 \mapsto 16 \quad 011 \mapsto 16
$$

Note that the sum of all occurrences is 47 although the function only has 32 output patterns. Algorithm $\mathrm{H}$ yields $\hat{\mu}(f)=16$ which corresponds to 4 additional output variables and therefore a total of 7 variables in a reversible embedding.

After applying Algorithm D (together with post compaction) one obtains the following equal PLA representation with no overlapping input cubes:

$$
\begin{array}{|lllll|lll|}
\hline 0 & 1 & - & 1 & - & 0 & 1 & 0 \\
0 & 0 & - & 1 & - & 0 & 1 & 1 \\
1 & - & - & - & - & 0 & 1 & 1 \\
\hline
\end{array}
$$

For this PLA representation Algorithm $\mathrm{H}$ will compute the following output pattern occurrences:

$$
000 \mapsto 8 \quad 010 \mapsto 4 \quad 011 \mapsto 20
$$

Since the PLA is representing a DSOP expression we have $\hat{\mu}(f)=\mu(f)=20$ which corresponds to 5 additional output variables and a total of 8 variables in a reversible embedding.

\section{BDD-based Approach}

All approaches presented thus far use a PLA representation in computing or approximating $\mu(f)$. However, sometimes such a representation is not available but the functions that are being considered can be represented as BDDs. In this section an algorithm is described that computes $\mu(f)$ directly on the BDD representation of an irreversible function $f \in \mathcal{B}_{n, m}$ in memory with $f\left(x_{1}, \ldots, x_{n}\right)=\left(y_{1}, \ldots, y_{m}\right)$. For this purpose, first the characteristic function $\chi_{f}$ is computed as described in (2).

For this purpose, the BDD of the characteristic function is constructed assuming the variable order $x_{1}<\cdots<x_{n}<$ $y_{1}<\cdots<y_{m}$. Then, let $V_{x}$ be the set of all vertices that are labeled $x_{i}$ for some $i$ and whose immediate parent is labeled $y_{j}$ for some $j$. The on-sets of the functions represented by each vertex in $V_{x}$ form a partition of all $2^{n}$ input patterns. This can be exploited by using the following proposition.

Proposition 4: In the BDD of $\chi_{f}$ every path from the start vertex to a vertex in $V_{x}$ visits all variables $y_{1}, \ldots, y_{m}$. Further, each vertex in $V_{x}$ has only one incoming edge.

Proof: Assume that there is a path from the start vertex to a vertex $v$ in $V_{x}$ in which a variable $v_{j}$ is not visited. Then, the monom represented by $v$ maps to more than one output in $f$ which contradicts that $f$ is a function. The same argument holds when we assume that $v$ has more than one incoming edge.

Now $\mu(f)$ can easily be computed using the vertices in $V_{x}$, i.e.:

$$
\mu(f)=\max \left\{\# \text { on }(\sigma(v)) \mid v \in V_{x}\right\}
$$

Example 7: The BDD for the characteristic function of the example in Fig. 3 is given in Fig. 7 Vertices from the set $V_{x}$ are filled in gray and annotated by the output pattern they are mapping to. Based on the paths one can count the minterms of each node which results in:

$$
100 \mapsto 4 \quad 101 \mapsto 9 \quad 010 \mapsto 8 \quad 001 \mapsto 6 \quad 000 \mapsto 5
$$

The numbers coincide with the results from Example 5.

\section{EMBEDding IRREVERSIBLE FunCTIONS}

In this section, we describe two approaches that construct a reversible embedding for a given irreversible function $f$. The first approach takes $f$ as a DSOP expression and guarantees a minimal number of additional lines. The second approach takes $f$ as a binary decision diagram and is heuristic. 


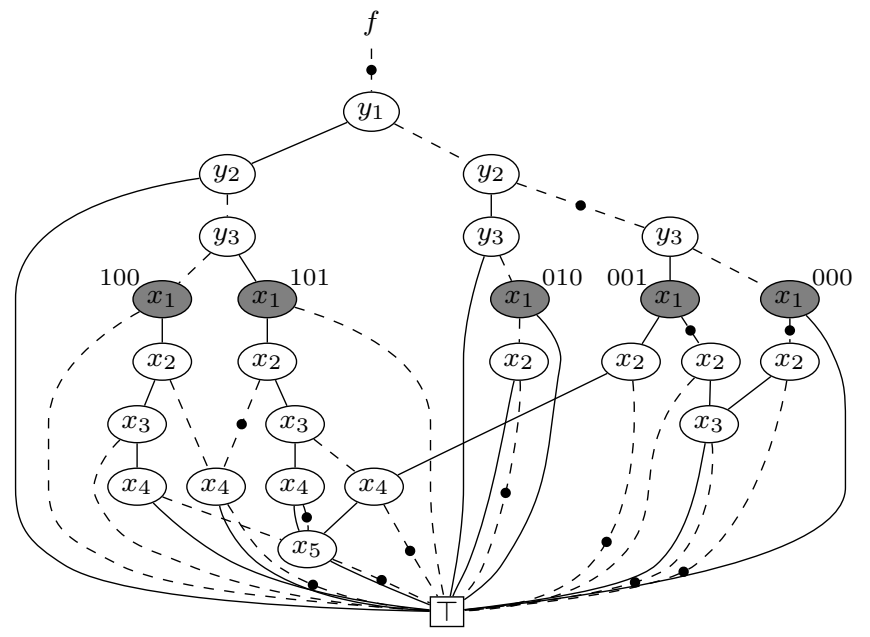

Fig. 7. BDD for the characteristic function of the example in Fig. 3

\section{A. Exact Cube-based Approach}

First, we will describe an exact embedding approach with respect to the number of additional lines that makes use of Algorithms $\mathrm{H}$ and D from the previous section. Given an irreversible function represented as a PLA, it is first transformed to represent a DSOP expression to determine the optimal number of additional lines. Then a reversible function is created by traversing all cubes of the DSOP expression. The algorithm requires the function to be represented as a DSOP expression in order to guarantee that no two input cubes have a non-empty intersection. Also, the algorithm creates a partial reversible function, i.e. not for all input patterns an output pattern is specified. However, all specified patterns in the reversible function are fully specified, i.e. they have no don't-care values.

We are making use of two helper functions in the following algorithm which are defined as follows.

Given a function $f \in \mathcal{B}_{n, m}$ and a set of output functions $o \in$ $\mathcal{P}(f)$, the function

$$
\operatorname{cube}(o) \stackrel{\text { def }}{=} \bigwedge_{i=1}^{m} \begin{cases}y_{i} & \text { if } f_{i} \in o, \\ \bar{y}_{i} & \text { otherwise }\end{cases}
$$

creates a cube that contains a positive literal $y_{i}$ if the function $f_{i}$ is contained in $o$ and a negative literal $\bar{y}_{i}$ otherwise.

Given a set of variables $x_{1}, \ldots, x_{n}$ the function

$$
\operatorname{inc}\left(x_{1}, \ldots, x_{n}\right)=\left(s_{1}, \ldots, s_{n}\right)
$$

computes the increment of the integer representation given by $x_{n} x_{n-1} \ldots x_{1}$, i.e.

$$
s_{i} \stackrel{\text { def }}{=} x_{i} \oplus \bigwedge_{j=1}^{i-1} x_{j} \quad \text { for } i \in\{1, \ldots, n\} .
$$

Note that both functions, cube and inc, are easy to implement using BDD manipulation. We are now ready to formulate the algorithm to embed a truth table based on a function's PLA representation.
Algorithm E (Cube-based Embedding). Given a function $f \in$ $\mathcal{B}_{n, m}$ represented as a DSOP, this algorithm generates a partial reversible function $g \in \mathcal{B}_{r, r}$ with

$$
g\left(\kappa_{1}, \ldots, \kappa_{p}, x_{1}, \ldots, x_{n}\right)=\left(y_{1}, \ldots, y_{m}, \gamma_{1}, \ldots, \gamma_{\ell(f)}\right)
$$

and $r=p+n=m+\ell(f)$ that embeds $f$ and is represented as its characteristic $\chi_{g}$ function using a BDD. Let $P_{f}=\left\{\left(c_{1}, o_{1}\right), \ldots,\left(c_{k}, o_{k}\right)\right\}$ be the PLA representation for the DSOP expression of $f$, i.e. no input cubes overlap.

E1. [Initialization.] Let $\chi_{g} \leftarrow \perp$ be a BDD with variable ordering

$$
\kappa_{1}<y_{1}<\kappa_{2}<y_{2}<\cdots<x_{n}<\gamma_{\ell(f)},
$$

i.e. inputs and outputs of $\chi_{g}$ appear in alternating order. Set $\ell \leftarrow \ell(f)$ where $\ell(f)=\left\lceil\log _{2} \mu(f)\right\rceil$ is obtained from Algorithm H. Further, set $j \leftarrow 0$ and CNT [o] $\leftarrow 0$ for $o \in$ $\mathcal{P}(f)$.

E2. [Loop over $C$.] If $j=k$, terminate. Otherwise, set $j \leftarrow$ $j+1, c \leftarrow c_{j}$, and $o \leftarrow o_{j}$.

E3. [Add cube to $\chi_{g}$.] Let

$$
\left(s_{1}, \ldots, s_{\ell}\right)=\operatorname{inc}^{q}\left(\gamma_{1}, \ldots, \gamma_{\ell}\right)
$$

where $q=\operatorname{CNT}[o]$, i.e., inc is applied $q$ times to the garbage outputs $\gamma_{1}, \ldots, \gamma_{\ell}$ and the values are stored in $s_{1}, \ldots, s_{\ell}$. Also, let

$$
\left\{d_{1}, \ldots, d_{t}\right\}=\operatorname{dc}(c) \quad \text { with } d_{1}<d_{2}<\cdots<d_{t}
$$

be the indexes of variables which are set don't-care in the input cube $c$. Let

$$
e=\underbrace{c}_{x^{\prime} \mathrm{s}} \wedge \underbrace{\operatorname{cube}(o)}_{y^{\prime} \mathrm{s}} \wedge \underbrace{\bigwedge_{i=1}^{p} \bar{\kappa}_{i}}_{\kappa^{\prime} \mathrm{s}} \wedge \underbrace{\bigwedge_{i=1}^{t}\left(x_{d_{i}} \leftrightarrow s_{i}\right) \wedge \bigwedge_{i=t+1}^{\ell} \bar{s}_{i}}_{\gamma^{\prime} \mathrm{s}}
$$

and set $\chi_{g} \leftarrow \chi_{g} \vee e$. Also, set CNT $[o] \leftarrow \operatorname{CNT}[o]+$ \# on $(c)$. Return to step 2.

In step 1 , an empty BDD $\chi_{g}$ is created that interleaves inputs and outputs in its variable ordering. The size of the BDD is determined by calculating the minimal number of additional lines $\ell(f)$ using Algorithm $\mathrm{H}$ after applying Algorithm D. The auxiliary array CNT is used to store how often an output pattern $o$ has been used and is initially initialized to 0 .

Step 2 manages the algorithm's loop over step 3. In each iteration one entry of $P_{f}$ is added to $g$. Step 3 creates a cube $e$ in (14) for the characteristic function $\chi_{g}$ based on the entry $(c, o)$ of the PLA representation $P_{f}$. This cube $e$ contains all required "ingredients," i.e. values for $x$ 's, $y$ 's, $\kappa$ 's, and $\gamma$ 's referring to inputs, outputs, constants, and garbage outputs, respectively.

- Input assignments for $x_{1}, \ldots, x_{n}$ are directly obtained from the input cube $c$.

- Output assignments for $y_{1}, \ldots, y_{m}$ are obtained from cube $(o)$.

- Constants $\kappa_{1}, \ldots, \kappa_{p}$ are all assigned 0 .

- The idea is to relate the values of the don't-care variables of $c$ to the garbage outputs $\gamma_{1}, \ldots, \gamma_{\ell}$. Since there may 
be equal output patterns, an offset $q=\operatorname{CNT}[o]$ is taken into account. To calculate the offset, the 'inc' function is applied to the $\gamma$ variables $q$ times. If there are less don't-care variables than garbage outputs, the remaining $s$ variables are inverted. Since $\ell$ is also obtained based on the number of don't-care variables in $c$, there are never more don't-care variables than garbage outputs in this step, i.e. $t \leq \ell$.

Example 8: We apply Algorithm E to the function with the PLA representation

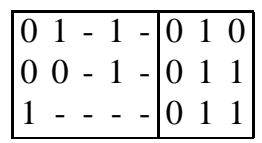

that has already been used in Section V-B Initially we set $\chi_{g} \leftarrow \perp$. Also we assign CNT $\left[\left\{f_{2}\right\}\right] \leftarrow 0$ for the first pattern and $\operatorname{CNT}\left[\left\{f_{2}, f_{3}\right\}\right] \leftarrow 0$ for the second and third pattern.

Since $\mu(f)=20$ we have $\ell(f)=5$ and therefore the reversible function $g \in \mathcal{B}_{8,8}$ maps inputs $\left(\kappa_{1}, \kappa_{2}, \kappa_{3}, x_{1}, x_{2}, x_{3}, x_{4}, x_{5}\right) \quad$ to outputs $\left(y_{1}, y_{2}, y_{3}, \gamma_{1}, \gamma_{2}, \gamma_{3}, \gamma_{4}, \gamma_{5}\right)$. For the first cube we have $\left(s_{1}, \ldots, s_{5}\right)=\left(\gamma_{1}, \ldots, \gamma_{5}\right)$ and hence we have

$$
e_{1}=\bar{\kappa}_{1} \bar{\kappa}_{2} \bar{\kappa}_{3} \bar{x}_{1} x_{2} x_{4} \bar{y}_{1} y_{2} \bar{y}_{3}\left(x_{3} \leftrightarrow \gamma_{1}\right)\left(x_{5} \leftrightarrow \gamma_{2}\right) \bar{\gamma}_{3} \bar{\gamma}_{4} \bar{\gamma}_{5}
$$

and we set CNT $\left[\left\{f_{2}\right\}\right] \leftarrow 4$. For the second cube we also have $\left(s_{1}, \ldots, s_{5}\right)=\left(\gamma_{1}, \ldots, \gamma_{5}\right)$ and hence we have

$$
e_{2}=\bar{\kappa}_{1} \bar{\kappa}_{2} \bar{\kappa}_{3} \bar{x}_{1} \bar{x}_{2} x_{4} \bar{y}_{1} y_{2} y_{3}\left(x_{3} \leftrightarrow \gamma_{1}\right)\left(x_{5} \leftrightarrow \gamma_{2}\right) \bar{\gamma}_{3} \bar{\gamma}_{4} \bar{\gamma}_{5}
$$

and we set CNT $\left[\left\{f_{2}, f_{3}\right\}\right] \leftarrow 4$. For the third cube we have $\left(s_{1}, \ldots, s_{5}\right)=\operatorname{inc}^{4}\left(\gamma_{1}, \ldots, \gamma_{5}\right)$, i.e.

$$
\begin{aligned}
s_{1} & =\gamma_{1} \\
s_{2} & =\gamma_{2} \\
s_{3} & =\gamma_{3} \oplus 1 \\
s_{4} & =\gamma_{4} \oplus \gamma_{3} \\
s_{5} & =\gamma_{5} \oplus \gamma_{3} \gamma_{4} .
\end{aligned}
$$

Hence, we have

$$
\begin{aligned}
e_{3} & =\bar{\kappa}_{1} \bar{\kappa}_{2} \bar{\kappa}_{3} x_{1} \bar{y}_{1} y_{2} y_{3}\left(x_{2} \leftrightarrow \gamma_{1}\right)\left(x_{3} \leftrightarrow \gamma_{2}\right) \\
& \wedge\left(x_{4} \leftrightarrow \bar{\gamma}_{3}\right)\left(x_{5} \leftrightarrow\left(\gamma_{4} \oplus \gamma_{3}\right)\right)\left(\bar{\gamma}_{5} \oplus \gamma_{3} \gamma_{4}\right)
\end{aligned}
$$

and update $\operatorname{CNT}\left[\left\{f_{2}, f_{3}\right\}\right] \leftarrow 20$. Overall, the partial reversible function embedding $f$ is given by $\chi_{g}=e_{1} \vee e_{2} \vee e_{3}$.

Correctness and completeness: Since the only loop in Algorithm E is bound by the number of cubes in the PLA representation, completeness is readily shown and it is left to show soundness.

Lemma 3: Algorithm E is sound.

Proof: To proof soundness we show that

(i) the input patterns are unique,

(ii) the output patterns are unique, and

(iii) $g$ embeds $f$.

Since the PLA represents a DSOP expression for $f$, it does not contain overlapping input cubes, and (i) holds trivially. Also (iii) follows immediately from (14). Only (ii) requires

\begin{tabular}{cc|cc}
\hline$c_{1} \ldots c_{m}$ & $x_{1} \ldots x_{n}$ & $y_{1} \ldots y_{m}$ & $\gamma_{1} \ldots \gamma_{n}$ \\
\hline $0 \ldots 00$ & $0 \ldots 0$ & $f_{1}(\vec{x}) \ldots f_{m}(\vec{x})$ & $0 \ldots 0$ \\
$\vdots$ & $\vdots$ & $\vdots$ & $\vdots$ \\
$0 \ldots 00$ & $1 \ldots 1$ & $f_{1}(\vec{x}) \ldots f_{m}(\vec{x})$ & $1 \ldots 1$ \\
$0 \ldots 01$ & $0 \ldots 0$ & $f_{1}(\vec{x}) \ldots \overline{f_{m}(\vec{x})}$ & $0 \ldots 0$ \\
$\vdots$ & $\vdots$ & $\vdots$ & $\vdots$ \\
$0 \ldots 01$ & $1 \ldots 1$ & $f_{1}(\vec{x}) \ldots \overline{f_{m}(\vec{x})}$ & $1 \ldots 1$ \\
$\vdots$ & $\vdots$ & $\vdots$ & $\vdots$ \\
$1 \ldots 11$ & $0 \ldots 0$ & $\overline{f_{1}(\vec{x})} \ldots \overline{f_{m}(\vec{x})}$ & $0 \ldots 0$ \\
$\vdots$ & $\vdots$ & $\vdots$ & $\vdots$ \\
$1 \ldots 11$ & $1 \ldots 1$ & $\overline{f_{1}(\vec{x})} \ldots \overline{f_{m}(\vec{x})}$ & $1 \ldots 1$ \\
\hline
\end{tabular}

Fig. 8. Bennett embedding scheme

some more thorough argument. We already motivated above that $t \leq \ell$ in step 3. Also since $\ell$ is obtained from Algorithm $\mathrm{H}$, one can see that CNT $[o] \leq \mathrm{MU}[o]$ is invariant. And since further $\ell \leq \log _{2} \mathrm{MU}[o]$, the assigned value for the garbage lines cannot "overlap."

\section{B. Heuristic BDD-based Embedding}

In this section, an approach is presented that embeds a function directly using BDDs. That is, the possibly costly way of having an PLA representation is omitted by directly starting from BDDs. These BDDs must be stored in memory and may have been created by any algorithm.

For this purpose, the idea of embedding as proposed by Bennett [1] who has initially proven the upper bound from Proposition 1 is adapted. In his constructive proof he already applied an explicit embedding which is known as Bennett Embedding and given as follows:

Theorem 1 (Bennett Embedding): Each function $f \in \mathcal{B}_{n, m}$ is embedded by the function $g \in \mathcal{B}_{m+n, m+n}$ such that

$$
g\left(\kappa_{1}, \ldots, \kappa_{m}, x_{1}, \ldots, x_{n}\right) \stackrel{\text { def }}{=}\left(y_{1}, \ldots, y_{m}, \gamma_{1}, \ldots, \gamma_{n}\right)
$$

with

$$
y_{i}\left(\kappa_{1}, \ldots, \kappa_{m}, x_{1}, \ldots, x_{n}\right)=\kappa_{i} \oplus f_{i}\left(x_{1}, \ldots, x_{n}\right)
$$

and

$$
\gamma_{i}\left(\kappa_{1}, \ldots, \kappa_{m}, x_{1}, \ldots, x_{n}\right)=x_{i} .
$$

Proof: The embedding is illustrated in Fig. 8 Assume $g$ is not injective, hence there is an output pattern that occurs at least twice. In particular, the function values for $\gamma_{i}$ must equal and according to (17) also the respective assignments for inputs $x_{i}$ must equal. But if the assignments for the inputs $x_{i}$ are the same, then the assignments for the inputs $\kappa_{j}$ must differ and due to (16) also the function values for $y_{i}$, contradicting our assumption.

Conducting the embedding posed by Theorem 1 on a truth table as illustrated in Fig. 8 is infeasible for large Boolean functions. Hence, we propose to perform this embedding directly using BDDs making use of the characteristic function. More precisely, given a function $f \in \mathcal{B}_{n, m}$, a characteristic 


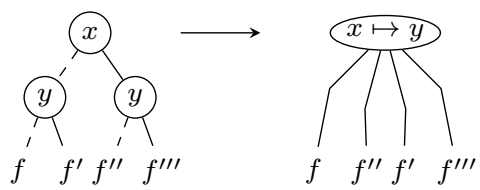

Fig. 9. Isomorphism between BDDs and QMDDs

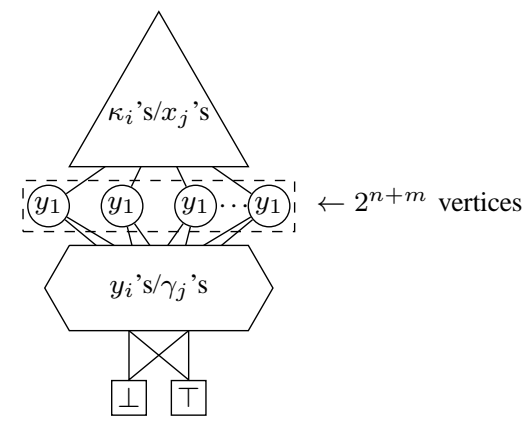

Fig. 10. Exponential size variable ordering

function $\chi_{g} \in \mathcal{B}_{2 m+2 n}$ that represents a function $g \in$ $\mathcal{B}_{m+n, m+n}$ according to Theorem 1 is computed by

$$
\begin{aligned}
\chi_{g}(\vec{\kappa}, \vec{x}, \vec{y}, \vec{\gamma}) & =\bigwedge_{i=1}^{m}\left(y_{i} \leftrightarrow\left(\kappa_{i} \oplus f_{i}\left(x_{1}, \ldots, x_{n}\right)\right)\right) \\
& \wedge \bigwedge_{i=1}^{n}\left(\gamma_{i} \leftrightarrow x_{i}\right)
\end{aligned}
$$

with $\vec{\kappa}=\kappa_{1}, \ldots, \kappa_{m}, \vec{x}=x_{1}, \ldots, x_{n}, \vec{y}=y_{1}, \ldots, y_{m}$, and $\vec{\gamma}=\gamma_{1}, \ldots, \gamma_{n}$ based on (3). As the experiments in the next section show, this enables the determination of an embedding for much larger functions.

Remark 2: If we construct a BDD from this function that follows the variable ordering

$$
\kappa_{1}<y_{1}<\cdots<\kappa_{m}<y_{m}<x_{1}<\gamma_{1}<\cdots<x_{n}<\gamma_{n}
$$

a graph results that is isomorphic to the QMDDs which are used for synthesis of large reversible functions in [19]. These QMDDs [10] are binary and use only Boolean values for the edge weights, therefore they represent permutation matrices. To illustrate the relations between BDD vertices for input and output variables of a characteristic function and a QMDD vertex consider Fig.9. The edges of a QMDD inherently represent an input output mapping which is explicitly expressed with a BDD for a characteristic function since it contains both input and output vertices. In the following, BDDs that represent characteristic functions of reversible functions are called RCBDDs. In fact, the algorithm for the QMDD-based synthesis presented in [19] can be performed on RC-BDDs instead.

The variable ordering that is interleaving input variables and output variables is not only necessary in order to directly synthesize the RC-BDD but also inevitable to keep the number of vertices small. More precisely, for each RC-BDD there exists two variable orderings which lead to an exponential number of vertices. In one of them all input variables are evaluated before all output variables (cf. Fig. 10). Since the RC-BDD represents a reversible function, each input pattern maps to a distinct output pattern. Hence, when all input patterns are evaluated first, $2^{m+n}$ vertices to represent all output patterns remain. Due to the reversibility, the same applies in case all output patterns are evaluated before all input patterns.

\section{EXPERIMENTAL EVALUATION}

We have implemented all algorithms that have been described in Sections $\mathrm{V}$ and $\mathrm{VI}$ in $\mathrm{C}++$ using RevKit [17] 3 This section presents the results of our evaluation. Benchmarks were taken from the LGSynth'93 benchmarks from Dmitri Maslov's benchmarks page 5 and from RevLib 6 The experimental evaluation has been carried out on a $3.4 \mathrm{GHz}$ Quad-Core Intel Xeon Processor with 32 GB of main memory running Linux 3.14. The timeout for all our experiments was set to 5000 seconds.

\section{A. Determining the Number of Additional Lines}

We have implemented the algorithms from Section $\nabla$ in the RevKit program 'calculate_required_lines' and evaluated them as follows. We have taken the benchmarks in PLA representation and approximated the number of lines using the heuristic cube-based approach (Section $\mathrm{V}$-A) . Afterwards we computed the exact number of additional lines using the exact cube-based approach (Section $\mathrm{V}-\mathrm{B}$ ) and by using the BDDbased approach (Section $\mathrm{V}-\mathrm{C}$ ). For the latter one the BDD was created from the PLA representation.

Table II list some selected experimental results. The first three columns list the name of the function together with its number of inputs and outputs. The fourth column lists the theoretical upper bound (Section IV-A). The remaining columns list the number of lines obtained by the three approaches. For the two approaches that compute the number of lines exactly, also the run-time is given. If no solution has been found in the given timeout, the cell is labeled with 'TO'. All results for the heuristic approach have been obtained in a few seconds. If the approximated result coincides with the exact one, it is emphasized using bold font. The benchmarks are sorted by their theoretical upper bound, i.e. the sum of the number of inputs and outputs.

The heuristic cube-based approach is often very close to the exact result. The highest measured difference in our experiments was 7 for the function add6. The function apex 4 represents the single case in which the approximated value is smaller than the exact one.

In case of the exact computation the cube-based and BDDbased approaches perform quite differently. For the BDDbased approach, the scalability seems to depend on the size of the function and hence may not scale for functions with more than 50 inputs and outputs. For some of the larger functions, the cube-based approach can still obtain a result, however, there are also smaller functions in which no solution can be found. This is probably because the scalability of the approach

\footnotetext{
${ }^{3}$ The source code that has been used to perform this evaluation is available at www.revkit.org (version 2.0).

4 www.cbl.ncsu.edu:16080/benchmarks/lgsynth93/

5 www.cs.uvic.ca/ dmaslov/

www.revlib.org
} 
TABLE II

EXPERIMENTS FOR DETERMINING THE NUMBER OF ADDITIONAL LINES

\begin{tabular}{|c|c|c|c|c|c|c|c|c|}
\hline Benchmark & $n$ & $m$ & Bennett & Heur. Cube & \multicolumn{2}{|c|}{ Exact Cube } & \multicolumn{2}{|c|}{ BDD } \\
\hline sym9 & 9 & 1 & 10 & 11 & 10 & 0.23 & 10 & 0.01 \\
\hline $\max 46$ & 9 & 1 & 10 & 10 & 10 & 0.03 & 10 & 0.01 \\
\hline sym10 & 10 & 1 & 11 & 11 & 11 & 1.72 & 11 & 0.02 \\
\hline wim & 4 & 7 & 11 & 10 & 9 & 0.00 & 9 & 0.00 \\
\hline z4 & 7 & 4 & 11 & 12 & 8 & 0.12 & 8 & 0.00 \\
\hline $\mathrm{z} 4 \mathrm{ml}$ & 7 & 4 & 11 & 12 & 8 & 0.06 & 8 & 0.00 \\
\hline sqrt8 & 8 & 4 & 12 & 13 & 9 & 0.09 & 9 & 0.00 \\
\hline $\mathrm{rd} 84$ & 8 & 4 & 12 & 11 & 11 & 0.17 & 11 & 0.01 \\
\hline root & 8 & 5 & 13 & 13 & 10 & 0.16 & 10 & 0.00 \\
\hline squar5 & 5 & 8 & 13 & 9 & 9 & 0.01 & 9 & 0.00 \\
\hline adr4 & 8 & 5 & 13 & 14 & 9 & 0.17 & 9 & 0.01 \\
\hline dist & 8 & 5 & 13 & 13 & 10 & 0.25 & 10 & 0.01 \\
\hline clip & 9 & 5 & 14 & 15 & 11 & 1.08 & 11 & 0.01 \\
\hline cm85a & 11 & 3 & 14 & 14 & 13 & 0.14 & 13 & 0.00 \\
\hline $\mathrm{pm} 1$ & 4 & 10 & 14 & 15 & 13 & 0.01 & 13 & 0.00 \\
\hline sao2 & 10 & 4 & 14 & 14 & 14 & 0.14 & 14 & 0.00 \\
\hline misex 1 & 8 & 7 & 15 & 15 & 14 & 0.01 & 14 & 0.00 \\
\hline $\operatorname{co} 14$ & 14 & 1 & 15 & 19 & 15 & 72.83 & 15 & 0.00 \\
\hline $\mathrm{dc} 2$ & 8 & 7 & 15 & 14 & 13 & 0.05 & 13 & 0.00 \\
\hline example2 & 10 & 6 & 16 & 16 & 14 & 1.36 & 14 & 0.01 \\
\hline inc & 7 & 9 & 16 & 14 & 14 & 0.01 & 14 & 0.00 \\
\hline mlp4 & 8 & 8 & 16 & 15 & 13 & 0.19 & 13 & 0.02 \\
\hline ryy6 & 16 & 1 & 17 & 19 & 17 & 157.65 & 17 & 0.00 \\
\hline $5 \times p 1$ & 7 & 10 & 17 & 17 & 10 & 0.10 & 10 & 0.02 \\
\hline parity & 16 & 1 & 17 & 16 & - & TO & 16 & 0.87 \\
\hline t481 & 16 & 1 & 17 & 19 & 17 & 1717.83 & 17 & 0.02 \\
\hline $\mathrm{x} 2$ & 10 & 7 & 17 & 19 & 16 & 0.05 & 16 & 0.00 \\
\hline sqr6 & 6 & 12 & 18 & 17 & 12 & 0.05 & 12 & 0.01 \\
\hline $\mathrm{dk} 27$ & 9 & 9 & 18 & 16 & 15 & 0.01 & 15 & 0.00 \\
\hline add6 & 12 & 7 & 19 & 20 & 13 & 46.12 & 13 & 0.10 \\
\hline $\mathrm{cmb}$ & 16 & 4 & 20 & 23 & 20 & 7.12 & 20 & 0.01 \\
\hline ex 1010 & 10 & 10 & 20 & 15 & 15 & 2.89 & 18 & 1.10 \\
\hline C7552 & 5 & 16 & 21 & 20 & 20 & 0.01 & 20 & 0.05 \\
\hline decod & 5 & 16 & 21 & 20 & 20 & 0.00 & 20 & 0.05 \\
\hline $\mathrm{dk} 17$ & 10 & 11 & 21 & 19 & 19 & 0.02 & 19 & 0.01 \\
\hline pcler8 & 16 & 5 & 21 & 23 & 21 & 28.11 & 21 & 0.00 \\
\hline tial & 14 & 8 & 22 & 23 & 19 & 1007.04 & 19 & 0.18 \\
\hline $\mathrm{cm} 150 \mathrm{a}$ & 21 & 1 & 22 & 22 & - & TO & 22 & 0.06 \\
\hline alu4 & 14 & 8 & 22 & 24 & 19 & 1270.29 & 19 & 0.13 \\
\hline apla & 10 & 12 & 22 & 22 & 22 & 0.03 & 22 & 0.01 \\
\hline $\mathrm{f} 51 \mathrm{~m}$ & 14 & 8 & 22 & 23 & 19 & 556.45 & 19 & 0.20 \\
\hline $\operatorname{mux}$ & 21 & 1 & 22 & 22 & - & TO & 22 & 0.14 \\
\hline cordic & 23 & 2 & 25 & 28 & - & TO & 25 & 0.06 \\
\hline $\mathrm{cu}$ & 14 & 11 & 25 & 26 & 25 & 0.02 & 25 & 0.00 \\
\hline in 0 & 15 & 11 & 26 & 25 & 25 & 1.46 & 25 & 0.05 \\
\hline 0410184 & 14 & 14 & 28 & 14 & 14 & 1227.84 & 14 & 7.40 \\
\hline apex4 & 9 & 19 & 28 & 25 & 26 & 0.95 & 26 & 23.95 \\
\hline misex 3 & 14 & 14 & 28 & 30 & 28 & 160.72 & 28 & 17.52 \\
\hline $\operatorname{misex} 3 c$ & 14 & 14 & 28 & 30 & 21 & 327.16 & 21 & 2.77 \\
\hline $\mathrm{cm} 163 \mathrm{a}$ & 16 & 13 & 29 & 31 & 25 & 625.43 & 25 & 0.01 \\
\hline frg1 & 28 & 3 & 31 & 32 & - & TO & 30 & 0.00 \\
\hline bw & 5 & 28 & 33 & 32 & 32 & 0.03 & 32 & 0.04 \\
\hline apex2 & 39 & 3 & 42 & 43 & - & TO & 42 & 5.14 \\
\hline pdc & 16 & 40 & 56 & 61 & 55 & 31.09 & - & TO \\
\hline spla & 16 & 46 & 62 & 65 & 61 & 32.72 & - & TO \\
\hline $\operatorname{ex} 5 p$ & 8 & 63 & 71 & 68 & 68 & 0.35 & - & TO \\
\hline seq & 41 & 35 & 76 & 76 & - & TO & - & TO \\
\hline cps & 24 & 109 & 133 & 136 & - & TO & - & TO \\
\hline apex 5 & 117 & 88 & 205 & 207 & - & TO & - & TO \\
\hline e64 & 65 & 65 & 130 & 129 & 129 & 0.07 & - & TO \\
\hline frg2 & 143 & 139 & 282 & 284 & - & TO & - & TO \\
\hline
\end{tabular}

depends on the number of cubes in the disjoint sum-of-product representation which does not directly depend on the function size.

\section{B. Cube-based Embedding}

We have implemented Algorithm E from Section VI-A in the RevKit program 'embed_pla' and evaluated it as follows.
TABLE III

EXPERIMENTS FOR EXACT CUBE-BASED EMBEDDING

\begin{tabular}{|c|c|c|c|c|c|}
\hline Benchmark & $n$ & $m$ & Lines & DSOP Comp. & Run-time \\
\hline sym9 & 9 & 1 & 1 & 0.23 & 0.22 \\
\hline $\max 46$ & 9 & 1 & 1 & 0.03 & 0.26 \\
\hline sym10 & 10 & 1 & 1 & 1.72 & 0.75 \\
\hline wim & 4 & 7 & 5 & 0.00 & 0.00 \\
\hline $\mathrm{z} 4$ & 7 & 4 & 1 & 0.12 & 0.01 \\
\hline $\mathrm{z} 4 \mathrm{ml}$ & 7 & 4 & 1 & 0.06 & 0.01 \\
\hline sqrt8 & 8 & 4 & 1 & 0.09 & 0.00 \\
\hline rd84 & 8 & 4 & 3 & 0.17 & 0.04 \\
\hline root & 8 & 5 & 2 & 0.16 & 0.01 \\
\hline squar5 & 5 & 8 & 4 & 0.01 & 0.00 \\
\hline adr4 & 8 & 5 & 1 & 0.17 & 0.02 \\
\hline dist & 8 & 5 & 2 & 0.25 & 0.01 \\
\hline clip & 9 & 5 & 2 & 1.08 & 0.06 \\
\hline cm85a & 11 & 3 & 2 & 0.14 & 0.30 \\
\hline pm1 & 4 & 10 & 9 & 0.01 & 0.00 \\
\hline sao2 & 10 & 4 & 4 & 0.14 & 0.10 \\
\hline $\operatorname{misex} 1$ & 8 & 7 & 6 & 0.01 & 0.00 \\
\hline co14 & 14 & 1 & 1 & 72.83 & 45.72 \\
\hline $\mathrm{dc} 2$ & 8 & 7 & 5 & 0.05 & 0.01 \\
\hline example2 & 10 & 6 & 4 & 1.36 & 0.15 \\
\hline inc & 7 & 9 & 7 & 0.01 & 0.00 \\
\hline mlp4 & 8 & 8 & 5 & 0.19 & 0.05 \\
\hline ryy6 & 16 & 1 & 1 & 157.65 & 101.18 \\
\hline $5 \times 1$ & 7 & 10 & 3 & 0.10 & 0.01 \\
\hline t481 & 16 & 1 & 1 & 1717.83 & 590.60 \\
\hline $\mathrm{x} 2$ & 10 & 7 & 6 & 0.05 & 0.04 \\
\hline sqr6 & 6 & 12 & 6 & 0.05 & 0.01 \\
\hline $\mathrm{dk} 27$ & 9 & 9 & 6 & 0.01 & 0.01 \\
\hline add6 & 12 & 7 & 1 & 46.12 & 3.89 \\
\hline $\mathrm{cmb}$ & 16 & 4 & 4 & 7.12 & 97.66 \\
\hline ex1010 & 10 & 10 & 5 & 2.89 & 6.07 \\
\hline C7552 & 5 & 16 & 15 & 0.01 & 0.08 \\
\hline decod & 5 & 16 & 15 & 0.00 & 0.09 \\
\hline $\mathrm{dk} 17$ & 10 & 11 & 9 & 0.02 & 0.22 \\
\hline pcler8 & 16 & 5 & 5 & 28.11 & 40.22 \\
\hline tial & 14 & 8 & 5 & 1007.04 & 40.28 \\
\hline alu4 & 14 & 8 & 5 & 1270.29 & 36.07 \\
\hline apla & 10 & 12 & 12 & 0.03 & 0.50 \\
\hline $\mathrm{f} 51 \mathrm{~m}$ & 14 & 8 & 5 & 556.45 & 39.20 \\
\hline $\mathrm{cu}$ & 14 & 11 & 11 & 0.02 & 0.75 \\
\hline in 0 & 15 & 11 & 10 & 1.46 & 24.99 \\
\hline 0410184 & 14 & 14 & 0 & 1227.84 & 1.36 \\
\hline apex4 & 9 & 19 & 17 & 0.95 & 38.89 \\
\hline misex3 & 14 & 14 & 14 & 160.72 & 768.98 \\
\hline $\operatorname{misex} 3 c$ & 14 & 14 & 7 & 327.16 & 144.87 \\
\hline $\mathrm{cm} 163 \mathrm{a}$ & 16 & 13 & 9 & 625.43 & 8.07 \\
\hline bw & 5 & 28 & 27 & 0.03 & 0.07 \\
\hline pdc & 16 & 40 & 39 & 31.09 & TO \\
\hline spla & 16 & 46 & 45 & 32.72 & TO \\
\hline ex5p & 8 & 63 & 60 & 0.35 & TO \\
\hline e64 & 65 & 65 & 64 & 0.07 & TO \\
\hline
\end{tabular}

We have taken those functions that did not lead to a timeout when determining the minimal number of lines using the exact cube-based approach in the previous section. Note that using that technique the DSOP expression needs to be computed before embedding it.

Table III list some selected experimental results. The first three columns list the name of the function together with its number of inputs and outputs. The fourth and fifth columns list the number of lines of the embedding together with the runtime required for computing the DSOP, respectively, which of course coincide with the numbers listed in Table [II The last column lists the run-time which is required for the embedding. The run-time for DSOP computation is not included in that time.

The run-time required for embedding the PLA is in most 
TABLE IV

LGSYNTH'93 BENCHMARK SUITE

\begin{tabular}{lrrrrr}
\hline Benchmark & $n$ & $m$ & Reading & Embedding & Run-time \\
\hline duke2 & 22 & 29 & 0.00 & 0.07 & 0.07 \\
misex3 & 14 & 14 & 0.04 & 0.14 & 0.18 \\
misex3c & 14 & 14 & 0.01 & 0.12 & 0.13 \\
spla & 16 & 46 & 0.07 & 0.38 & 0.45 \\
e64 & 65 & 65 & 0.00 & 0.20 & 0.20 \\
apex2 & 36 & 3 & 0.12 & 0.39 & 0.51 \\
pdc & 16 & 40 & 0.08 & 0.62 & 0.70 \\
seq & 41 & 35 & 0.15 & 0.70 & 0.85 \\
cps & 24 & 109 & 0.02 & 0.53 & 0.55 \\
apex1 & 45 & 45 & 0.25 & 1.23 & 1.48 \\
apex5 & 117 & 88 & - & - & TO \\
ex4p & 128 & 28 & - & - & TO \\
\hline
\end{tabular}

of the cases less compared to the time required for computing the DSOP with the exception of some few cases. For the four largest functions in this requirement the embedding algorithm leads to a time-out although the DSOP could be computed efficiently.

\section{BDD-based Embedding}

This section summarizes the results from three different experiments that we implemented and performed in order to evaluated the BDD-based embedding that has been described in Section VI-B.

1) LGSynth'93 Benchmarks: In a first experiment, the algorithm is applied to all 37 functions of the LGSynth'93 benchmark suite. Since the functions are represented as PLA in this case, we have added an option to the RevKit program 'embed_pla' to choose between the exact cube-based and heuristic BDD-based embedding. Table IV lists the results for the hardest instances, i.e. the instances which required the largest run-time. The first three columns of the table list the name of the benchmark, the number of input variables $n$, and the number of output variables $m$. The remaining columns list run-times in seconds for reading the benchmark and embedding it as well as the total run-time. Except for two functions that could not be processed due to memory limitations, the algorithm has no problems with handling these functions. As a result, efficient embeddings for them have been determined for the first time. The largest function $c p s$ involves 131 inputs and outputs. No more than $8 \mathrm{CPU}$ seconds are required to obtain a result.

In order to underline the importance of the variable ordering as discussed in Section [V] we have repeated the same experiment by keeping the natural variable ordering

$\kappa_{1}<\cdots<\kappa_{m}<x_{1}<\cdots<x_{n}<g_{1}<\cdots<g_{m}<\gamma_{1}<\cdots<\gamma_{n}$.

In this case, 22 of the 37 functions could not have been processed due to memory limitations. For the remaining functions, an embedding was determined. However, this included only rather small functions.

2) 2-level Redundancy Functions: Besides predefined functions that are read in from a file, additional experiments have been carried out in which the BDDs have been created using manipulation operations in the BDD package itself. For this purpose, BDDs representing functions which are applied in
TABLE V

2-LEVEL REDUNDANCIES FUNCTIONS

\begin{tabular}{llrrr}
\hline Rows $p$ & Columns $q$ & $n$ & $m$ & Run-time \\
\hline 5 & 5 & 30 & 1 & 0.06 \\
6 & 6 & 42 & 1 & 0.79 \\
7 & 7 & 56 & 1 & 6.80 \\
8 & 8 & 72 & 1 & 77.98 \\
9 & 9 & 90 & 1 & 1057.56 \\
10 & 10 & 110 & 1 & 9615.86 \\
11 & 10 & 112 & 1 & MO \\
10 & 11 & 120 & 1 & MO \\
11 & 11 & 132 & 1 & MO \\
\hline
\end{tabular}

fault tolerant systems have been considered. More precisely, let $p, q \in \mathbb{N}$, then given variables $x_{i}$ and $y_{i j}$ for $i=1, \ldots, p$ and $j=1, \ldots, q$, the Boolean function

$$
f=\bigwedge_{j=1}^{q} \bigvee_{i=1}^{p} x_{i} \wedge y_{i j}
$$

is a 2-level redundancy function [12]. Such functions encode cascade redundancies in critical systems and can be found in formal methods for risk assessment [13]. Further, the function $f$ is true if and only if all columns of the matrix product

$$
x \cdot Y=\left(x_{1} x_{2} \ldots x_{p}\right)\left(\begin{array}{cccc}
y_{11} & y_{12} & \ldots & y_{1 q} \\
y_{21} & y_{22} & \ldots & y_{2 q} \\
\vdots & \vdots & \ddots & \vdots \\
y_{p 1} & y_{p 2} & \ldots & y_{p q}
\end{array}\right)
$$

are positive, i.e. when the rows of $Y$ selected by $x$ cover every column of that matrix [7].

Table $\mathrm{V}$ shows the results for this experiment that have been generated using the RevKit test-case 'redundancy_functions'. The columns list the values for $p$ and $q$, the resulting number of inputs $n$ and outputs $m$ for the corresponding BDD, as well as the run-time required for the embedding. It can be seen that the algorithm terminates within a reasonable amount of time for BDDs with up to 100 variables. However, if more variables are considered, the BDDs became too large and the algorithm ran into memory problems. Clearly, the efficiency of the algorithm highly depends on the size of the BDDs. Nevertheless, also for this set of large functions, efficient embeddings have been obtained.

3) Restricted Growth Sequences: Similarly, another experiment has been conducted on functions representing restricted growth sequences which should be embedded as reversible functions. More precisely, a permutation $\{1, \ldots, p\}$ into disjoint subsets can efficiently be represented by a string sequence $a_{1}, \ldots, a_{p}$ of non-negative integers such that $a_{1}=0$ and

$$
a_{j+1} \leq 1+\max \left(a_{1}, \ldots, a_{j}\right) \text { for } 1 \leq j<p .
$$

This sequence is called a restricted growth sequence and elements $j$ and $k$ belong to the same subset of the partition if and only if $a_{j}=a_{k}$ [6], [7].

Table VI lists the results when applying the heuristic embedding algorithm to BDDs representing these restricted 
TABLE VI

RESTRICTED GROWTH SEQUENCES

\begin{tabular}{lrrr}
\hline Sequence length $p$ & $n$ & $m$ & Run-time \\
\hline 5 & 15 & 1 & 0.00 \\
10 & 55 & 1 & 0.02 \\
15 & 120 & 1 & 0.17 \\
20 & 210 & 1 & 0.86 \\
25 & 325 & 1 & 3.04 \\
30 & 465 & 1 & 9.13 \\
35 & 630 & 1 & 23.80 \\
40 & 820 & 1 & 60.26 \\
45 & 1035 & 1 & 139.71 \\
50 & 1275 & 1 & 295.93 \\
55 & 1540 & 1 & 566.00 \\
60 & 1830 & 1 & 1029.67 \\
65 & 2145 & 1 & 1802.98 \\
70 & 2485 & 1 & 2966.90 \\
75 & 2850 & 1 & 4811.23 \\
80 & 3240 & 1 & TO \\
\hline
\end{tabular}

growth sequences for different sequence lengths $p$. The experiment has been implemented in the RevKit test-case ' $r e$ stricted_growth_sequence'. The columns list the length, number of inputs and outputs, and the total run-time in seconds. It can be seen that here even functions with more than 400 variables can be handled within a reasonable amount of run-time.

\section{CONCLUSIONS}

Significant progress has been made in the synthesis of reversible circuits. In particular, scalability has intensively been addressed. However, no solution was available thus far that embeds large irreversible functions into reversible ones. In this work, we have investigated this problem extensively. We showed that this problem is coNP-hard and thus intractable. We then described approaches both for determining the number of lines and for embedding an irreversible function. Sumof-products and binary decision diagrams have been used as function representations in these approaches and also both exact approaches and heuristics have been presented. For the first time, this enabled the determination of compact embeddings of functions containing hundreds of variables.

Future work includes the application of the proposed embedding scheme to scalable synthesis approaches for reversible functions for which thus far no embedding has been available [18], [19]. Further, there are some interesting open problems that resulted from the research presented in this paper:

1) It would be good to have an approach for approximating the number of additional lines which guarantees not to give an under approximation.

2) It is interesting whether one can find an embedding for a general PLA representation, which may contain overlapping input cubes.

3) An exact embedding approach based on the exact BDDbased method for determining the minimal number of additional lines would allow for an embedding method that can work directly on BDDs and does not necessarily require a PLA representation.

Overall, an important open problem in reversible circuit synthesis has been solved by providing solutions to embed large irreversible functions. Also, many interesting new open problems are posed for future research on this topic.

\section{ACKNOWLEDGMENTS}

The authors wish to thank Stefan Göller for many interesting discussions and his contribution in proving the lower bounds for the embedding problem.

\section{REFERENCES}

[1] C. H. Bennett. Logical reversibility of computation. IBM Journal of Research and Development, 17(6):525-532, 1973.

[2] A. Bernasconi, V. Ciriani, F. Luccio, and L. Pagli. Compact DSOP and partial DSOP forms. Theory Comput. Syst., 53(4):583-608, 2013.

[3] R. E. Bryant. Graph-based algorithms for Boolean function manipulation. IEEE Trans. on Computers, 35(8), 1986.

[4] A. De Vos and Y. Van Rentergem. Young subgroups for reversible computers. Advances in Mathematics of Communications, 2(2):183200, 2008.

[5] D. Große, R. Wille, G. W. Dueck, and R. Drechsler. Exact multiplecontrol Toffoli network synthesis with SAT techniques. IEEE Trans. on $C A D, 28(5): 703-715,2009$.

[6] G. Hutchinson. Partioning algorithms for finite sets. Communications of the ACM, 6(10):613-614, 1963.

[7] D. E. Knuth. The Art of Computer Programming, volume 4A. AddisonWesley, Upper Saddle River, New Jersey, 2011.

[8] C.-C. Lin and N. K. Jha. RMDDS: Reed-Muller decision diagram synthesis of reversible logic circuits. Journal on Emerging Technologies in Computing Systems, 10(2):14, 2014.

[9] D. M. Miller, D. Maslov, and G. W. Dueck. A transformation based algorithm for reversible logic synthesis. In Design Automation Conference, pages 318-323, 2003.

[10] D. M. Miller and M. A. Thornton. QMDD: A decision diagram structure for reversible and quantum circuits. In Int'l Symp. on Multiple-Valued Logic, page 30, 2006.

[11] D. M. Miller, R. Wille, and G. Dueck. Synthesizing reversible circuits for irreversible functions. In EUROMICRO Symp. on Digital System Design, pages 749-756, 2009.

[12] M. Nikolskaia and L. Nikolskaia. Size of OBDD representation of 2level redundancies functions. Theoretical Computer Science, 255(12):615-625, 2001.

[13] M. Nikolskaia and A. Rauzy. Heuristics for BDD handling of sumof-products formulae. In European Saftety and Reliability Association Conference, pages 1459-1465, 1998.

[14] B. Padmanabhan and D. Edwards. Self-timed realization of combinational logic. In Int'l Workshop on Logic Synthesis, 2010.

[15] C. E. Shannon. A symbolic analysis of relay and switching circuits. Trans. American Institute of Electrical Engineers, 57(38-80):713-723, 1938.

[16] V. Shende, A. Prasad, I. Markov, and J. Hayes. Synthesis of reversible logic circuits. IEEE Trans. on CAD, 22(6):710-722, 2003.

[17] M. Soeken, S. Frehse, R. Wille, and R. Drechsler. RevKit: An open source toolkit for the design of reversible circuits. Lecture Notes in Computer Science, 7165:64-76, 2012. Selected Papers from the Third International Workshop on Reversible Computation.

[18] M. Soeken, L. Tague, G. W. Dueck, and R. Drechsler. Ancilla-free synthesis of large reversible functions using binary decision diagrams. 2014. submitted.

[19] M. Soeken, R. Wille, C. Hilken, N. Przigoda, and R. Drechsler. Synthesis of reversible circuits with minimal lines for large functions. In Asia and South Pacific Design Automation Conference, pages 85-92, 2012.

[20] R. Wille and R. Drechsler. BDD-based synthesis of reversible logic for large functions. In Design Automation Conference, pages 270-275, 2009.

[21] R. Wille, O. Keszöcze, and R. Drechsler. Determining the minimal number of lines for large reversible circuits. In Design, Automation and Test in Europe, 2011.

[22] R. Wille, M. Soeken, and R. Drechsler. Reducing the number of lines in reversible circuits. In Design Automation Conference, pages 647-652, 2010. 